International Journal of Modern Physics B

(C) World Scientific Publishing Company

\title{
Positional order of the pinned Abrikosov flux-line lattice in samples of finite thickness
}

\author{
A.M. Ettouhami \\ Department of Physics, University of Florida, P.O. Box 118440, Gainesville, FL 32606, USA \\ mouneim@phys.ufl.edu
}

\begin{abstract}
We study translational correlations of the vortex center of mass positions of the Abrikosov flux-line lattice in superconducting samples of finite thickness $L$ (along the direction of flux-lines). The Larkin correlation lengths for the center of mass mode of the flux lines in the presence of point and correlated disorder are computed, and we find that in the case of point disorder the average (i.e. center of mass) position of flux lines maintains positional order on length scales which scale like $\sim \sqrt{L}$ in $2+1$ dimensions. On still longer length scales, however, we find using a replica Gaussian variational approach that center of mass correlations cross over to a power law growth of the form $r_{\perp} / L$, which should be observable in superconducting thin films.
\end{abstract}

Keywords: Flux-line lattice, Pinning, Long range order.

\section{Introduction}

There has been a great interest in the properties of the Abrikosov flux-line lattice ${ }^{1}$ (FLL) in high temperature superconductors ${ }^{2}$ (HTSCs) during the past eighteen years. This interest was motivated by the remarkable physical properties of these materials, such as their high superconducting critical temperatures and their anisotropic, layered structure, with both properties dramatically increasing the importance of thermal fluctuations. Thus, it has quickly been recognized that HTSCs are an excellent system for studying the combined effects of thermal fluctuations and quenched disorder on flux-line assemblies. These combined effects lead to a remarkably rich phase diagram for the flux-line system, the phenomenology of which, even after many years of experimental and theoretical investigations, continues to pose many exciting and challenging questions. ${ }^{3,4}$

In contrast to the usual phase diagram of conventional, low temperature superconductors, which shows an Abrikosov flux-line lattice on the whole region $H_{c 1}(T)<H<H_{c 2}(T)$ delimited by the lower and upper critical fields, $H_{c 1}(T)$ and $H_{c 2}(T)$ respectively (Fig. 1), because thermal fluctuations are now stronger, the vortex lattice melts into a flux-line liquid over a significant region of the magnetic field $(H)$-temperature $(T)$ phase diagram (Fig. 2, upper panel).

In the presence of disorder, most theoretical studies of flux pinning in HTSCs 


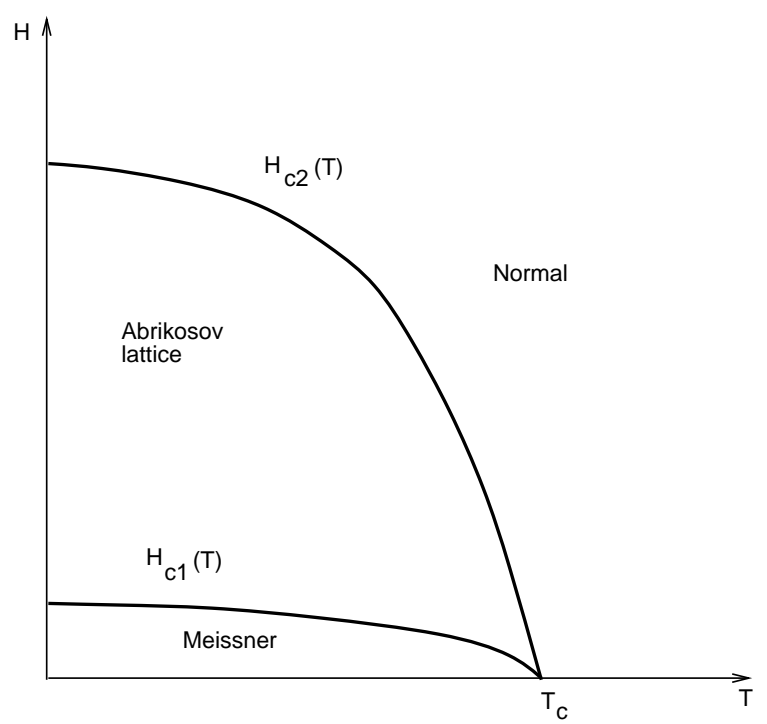

Fig. 1. Phase diagram of the flux line system in conventional (low-temperature) superconductors. Below $H_{c 1}(T)$, the superconductor is in the so-called Meissner state, characterized by perfect diamagnetism, while above $H_{c 2}(T)$ the system becomes normal (no superconductivity).

have concentrated on trying to understand nature of translational and orientational order of flux-lines as a function of temperature and applied external field, and the implications of the possible existence of glassy phases on the nonequilibrium dynamics, especially in the presence of an external current. These studies also led to an investigation of these questions in the more general context of elastic media in the presence of thermal fluctuations, pinning (random and periodic) and external drive, stimulated by strong connections to other mathematically related condensed matter systems such as charge density waves (CDWs), Wigner crystals, domain walls, crystal surfaces, etc. For the particular case of flux-lines in presence of disorder, the phase diagram turns out to be a very rich one, with the flux-line lattice losing true translational long range order (LRO) and exhibiting a highly nonlinear flux flow resistivity. For weak disorder and low applied magnetic fields, this is a phase with quasi long range order (QLRO) and no topological defects which has been termed Bragg glass. At higher external fields, the Bragg glass leads way to a vortex glass with dislocations that improve the benefit from pinning energy (Fig. 2, lower panel).

On the historical level, the first and decisive step for studying the pinning problem using statistical mechanics was done in the remarkable work of Larkin ${ }^{5,17}$, where it was recognized that pinning can be treated perturbatively inside finite size domains (the Larkin domains) of coherently pinned flux lines, whose spatial extent has become known as the Larkin length $R_{c}$. Beyond this length, the disorder Hamiltonian has a large number of low lying metastable "ground" states, which dooms 

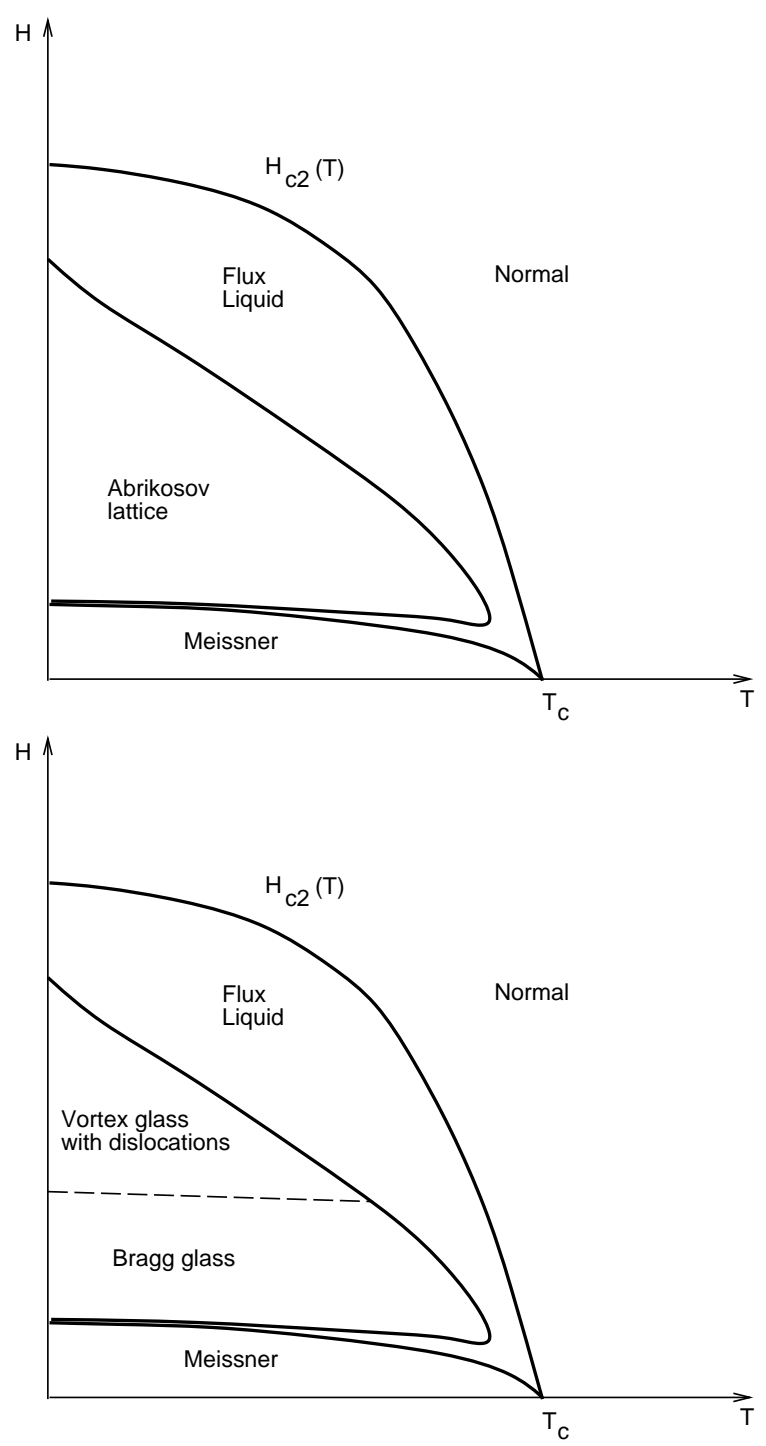

Fig. 2. Phase diagram of the flux line system in high temperature superconductors. Upper panel: in absence of disorder. Lower panel: in presence of disorder.

to eventual failure all direct methods based on straightforward perturbation theory. Therefore, new, more sophisticated methods had to be invented to treat the pinning problem beyond the Larkin length $R_{c}$.

The key quantity characterizing LRO in disordered elastic media is the roughness

$$
C(\mathbf{r})=\overline{\left\langle[\mathbf{u}(\mathbf{r})-\mathbf{u}(\mathbf{0})]^{2}\right\rangle},
$$

where $\mathbf{u}(\mathbf{r})$ is the displacement of a vortex from its undistorded position $\mathbf{r}$, and where the angular brackets denote averaging over thermal fluctuations and the over- 
bar denotes averaging over the quenched disorder. Within a single Larkin correlated volume, one can neglect the dependence of the random pinning force $\mathbf{f}(\mathbf{u}, \mathbf{r})$ experienced by flux lines on the displacements $\mathbf{u}$. Simple analysis ${ }^{5}$ (see below) shows that $C(r) \sim r^{2 \zeta}$ with the so-called wandering or roughness exponent $\zeta=(4-d) / 2(d$ here is the dimension of the lattice). Beyond the Larkin length $R_{c}$, we cannot neglect the dependence of $\mathbf{f}(\mathbf{u}, \mathbf{r})$ on the displacements $\mathbf{u}$, and the periodicity of the flux line system has also to be taken into account. Following the pioneering work of Larkin (and analogous works by Lee and Rice ${ }^{7}$ in the context of CDWs and by Imry and $\mathrm{Ma}^{6}$ in the context of random ferromagnets), significant progress in understanding the physics on scales longer than $R_{c}$ was achieved thanks to the contributions of several authors, such as Villain and Fernandez ${ }^{8}$, D.S. Fisher ${ }^{9}$, Nattermann ${ }^{10}$, Bouchaud, Mézard and Yedidia ${ }^{11}$ and Korshunov ${ }^{12}$. These results were then fruitfully applied to the flux line lattice problem where it was shown by Giamarchi and Le Doussal ${ }^{13}$ (using a variant of the replica variational method for elastic manifolds of Mézard and Parisi ${ }^{14}$ ) that at length scales longer than $R_{c}$ the flux array is characterized by a logarithmic growth of flux line displacements

$$
C(\mathbf{r}) \sim \ln \left(r / R_{c}\right),
$$

both in two and three dimensions, a result which has been confirmed by an independent, functional renormalization group (FRG) calculation. ${ }^{15}$

An issue which is not sufficiently appreciated in the literature at the present time is the fact that the phonon field $\mathbf{u}(\mathbf{r})=\mathbf{u}(\mathbf{x}, z)$ includes all the internal modes of the flux lines, and that positional order is in fact best characterized by the displacement of the center of mass (CM) positions of vortices, which represent the average positions of the flux-lines. Indeed, flux-line lattices are essentially twodimensional, as exhibited by the fact that both direct and reciprocal lattice vectors of the FLL lie in two-dimensional space. This is an essential difference between vortex lattices and ordinary three-dimensional solids, which implies in particular that in order to characterize the displacement of the average position of a given flux-line from its equilibrium position $\mathbf{R}_{i}$, it is enough to consider the displacement $\mathbf{u}_{0 i}=\mathbf{r}_{0 i}-\mathbf{R}_{i}$ of the vortex center of mass $\mathbf{r}_{0 i}$. Hence, to find the displacement of the average (i.e. center of mass) position of vortices, we need to consider the correlator

$$
C_{0}(\mathbf{r})=\overline{\left\langle\left[\mathbf{u}_{0}(\mathbf{r})-\mathbf{u}_{0}(\mathbf{0})\right]^{2}\right\rangle} .
$$

Although the above distinction between the general correlator $C(\mathbf{r})$ of Eq. (1) and the $\mathrm{CM}$ correlator $C_{0}(\mathbf{r})$ is of no great importance and only of academic interest for pure (unpinned) flux line lattices in three dimensions, below we will show that proper handling of the CM mode of flux lines alters the asymptotic behavior of the translational correlation function (1) in the presence of disorder. In order to do so, we shall extend the replica Gaussian variational method of reference ${ }^{13}$ to the case of samples of finite thickness $L_{z}$ along the direction of flux lines, being careful to separate the CM mode from the internal modes of flux lines. 
Our main result is that for the fluctuations of the center of mass positions of flux lines the logarithmic roughness of Eq. (2) should be replaced, in finite size systems (and especially in thin films) by the following algebraic growth of flux-line fluctuations (here the vortices are directed along the $\hat{\mathbf{z}}$ axis, and $\mathbf{r}_{\perp}=x \hat{\mathbf{x}}+y \hat{\mathbf{y}}$ )

$$
C_{0}\left(r_{\perp}\right) \sim \frac{r_{\perp}}{L_{z}} .
$$

Indeed, the main thrust of the argument made in ref. 13 relied on the fact that the elastic propagator $G(q)$ in presence of disorder had a wavevector dependence of the form

$$
G(q) \propto \frac{1}{q^{d}}
$$

in $d$ dimensions for $2<d<4$, hence the result (2). It is important to realize that the elastic propagator in Eq. (5) is valid, strictly speaking, for three-dimensional solids (although it has been derived in the context of vortex lines). If we recall that $q^{2}=q_{\perp}^{2}+q_{z}^{2}$ (with $\mathbf{q}_{\perp}$ and $q_{z}$ the wavevectors along the directions perpendicular and parallel to the vortices, respectively), then it is easy to see from the above result that the elastic propagator $G_{0}(q)$ for the $\mathrm{CM}\left(q_{z}=0\right)$ mode is given by:

$$
G_{0}\left(q_{\perp}\right) \propto \frac{1}{q_{\perp}^{d}}
$$

which directly leads to Eq. (4) in $d=3$ dimensions. While we argue that the above result, Eq. (4), is in principle valid for samples of arbitrary thickness, it is mostly relevant to thin superconducting films with a small enough thickness $L_{z}$ compared to their size $L_{\perp}$ in transverse directions (as long as $L_{z} \gg \xi$, where $\xi$ is the coherence length along the direction of flux lines, so that flux lines have both $\mathrm{CM}$ and internal fluctuations). In such films, the above equation can be of direct experimental relevance and will lead to the complete destruction of translational long range order on length scales $r_{\perp}>L_{z}$.

The rest of this paper is organized as follows. In Sec. 2, we shall start our investigation by carefully defining the $\mathrm{CM}$ and internal degrees of freedom of the flux lines and the associated phonon fields. We shall then briefly discuss the case of disorder-free flux line lattices in $2+1$ and $1+1$ dimensions. In Sec. 3, we shall consider the effect of an external pinning potential, both perturbatively, and using a replica Gaussian variational approach. In Sec. 4, we briefly comment on positional order for the CM mode for moving flux line arrays in disorder, and in Sec. 5 we will present our conclusions.

\section{Elasticity in the center of mass representation}

In order to fix ideas, let us consider a $d$-dimensional superconducting sample in an applied magnetic field $\mathbf{H}=H \hat{\mathbf{z}}$. The sample thickness in the $z$ direction will be denoted by $L$. Vortex trajectories will be parametrized by the $d$-dimensional vector $\mathbf{r}_{i}(z)=\left(\mathbf{x}_{i}(z), z\right)$, where $\mathbf{x}_{i}(z)$ denotes the transverse position of the $i$ th flux line at 
height $z$, and the number of transverse dimensions will be denoted by $d_{\perp}=(d-1)$. We shall introduce the following decomposition of $\mathbf{x}_{i}(z)$ in Fourier modes ${ }^{18,19}$

$$
\mathbf{x}_{i}(z)=\sum_{n=-\infty}^{\infty} \mathbf{x}_{i}\left(q_{n}\right) \mathrm{e}^{i \mathbf{q}_{n} z},
$$

where $q_{n}=2 n \pi / L$ and where the Fourier components $\mathbf{x}\left(q_{n}\right)$ are related to $\mathbf{x}_{i}(z)$ by

$$
\mathbf{x}\left(q_{n}\right)=\frac{1}{L} \int_{0}^{L} d z \mathbf{x}_{i}(z) \mathrm{e}^{i q_{n} z},
$$

as can be verified by using the orthogonality relation:

$$
\int_{0}^{L} d z \mathrm{e}^{i q_{n} z}\left(\mathrm{e}^{i q_{m} z}\right)^{*}=L \delta_{n, m} .
$$

The above Fourier decomposition is similar to the decomposition of internal modes into Rouse modes commonly used in polymer physics ${ }^{18}$. For the developments that will follow, it will prove useful to write $\mathbf{x}_{i}(z)$ in the form

$$
\mathbf{x}_{i}(z)=\mathbf{x}_{0 i}+\mathbf{u}_{1 i}(z),
$$

where

$$
\mathbf{x}_{0 i}=\frac{1}{L} \int_{0}^{L} d z \mathbf{x}_{i}(z)
$$

is the position of the center of mass of the $i$ th flux line, while (here c.c. denotes complex conjugation)

$$
\mathbf{u}_{1 i}(z)=\sum_{n=1}^{\infty}\left\{\mathbf{x}\left(q_{n}\right) \mathrm{e}^{i q_{n} z}+\text { c.c. }\right\}
$$

is the displacement of the flux-line at height $z$ with respect to the CM position $\mathbf{x}_{0 i}$. Note that this last quantity itself is a dynamical variable, since the CM position $\mathbf{x}_{0 i}$ of the $i$-th flux line itself fluctuates around its ideal lattice position $\mathbf{X}_{i}$. We thus see that the displacement of the flux line at height $z$ with respect to its equilibrium position $\mathbf{X}_{i}$ is given by

$$
\begin{aligned}
\mathbf{u}_{i}(z) & =\mathbf{x}_{i}(z)-\mathbf{X}_{i}, \\
& =\mathbf{u}_{0 i}+\mathbf{u}_{1 i}(z),
\end{aligned}
$$

where the $z$-independent quantity $\mathbf{u}_{0 i}=\mathbf{x}_{0 i}-\mathbf{X}_{i}$ is the displacement of the center of mass position with respect to the equilibrium position $\mathbf{X}_{i}$ (see figure 3 ).

In order to be able to define mathematically tractable models, one has to perform a continuum limit, whereby the individual displacement fields $\left\{\mathbf{u}_{i}(z)\right\}$ are replaced by a smooth interpolating function $\mathbf{u}(\mathbf{x}, z)$. The displacement field $\mathbf{u}(\mathbf{x}, z)$ will be decomposed in the following Fourier modes: ${ }^{20}$

$$
\mathbf{u}(\mathbf{x}, z)=\sum_{n=-\infty}^{\infty} \int_{\mathbf{q}} \mathbf{u}\left(\mathbf{q}, q_{n}\right) \mathrm{e}^{i\left(\mathbf{q} \cdot \mathbf{x}+q_{n} z\right)},
$$




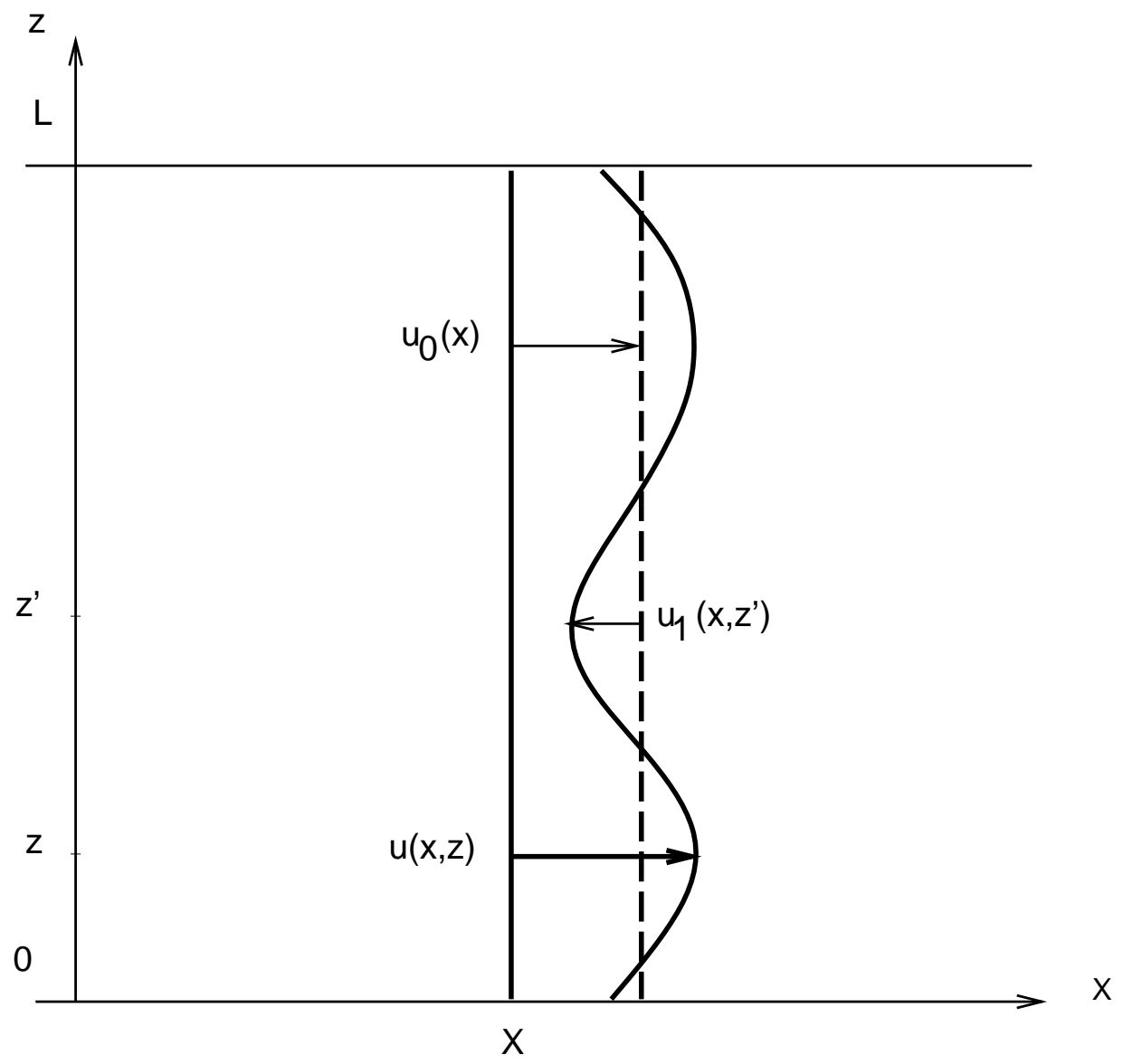

Fig. 3. Definition of the center of mass and internal modes. The solid and dashed vertical lines indicate the location of the equilibrium and center of mass positions, respectively. $\mathbf{u}_{0}(\mathbf{x})$ is the displacement of the center of mass position with respect to the ideal position $\mathbf{x} . \mathbf{u}_{1}(\mathbf{x}, z)$ is the displacement of the flux line with respect to the center of mass position at height $z$, and $\mathbf{u}(\mathbf{x}, z)$ is the diplacement of the flux line at height $z$ with respect to its equilibrium position. One has $\mathbf{u}(\mathbf{x}, z)=\mathbf{u}_{0}(\mathbf{x})+\mathbf{u}_{1}(\mathbf{x}, z)$.

where $\int_{\mathbf{q}}$ stands for $\int_{B Z} d^{d_{\perp}} \mathbf{q} /(2 \pi)^{d_{\perp}}$ (note that $\mathbf{q}$ here is a $d_{\perp}$-dimensional vector), and the integration is over the first Brillouin zone of the flux line lattice. The Fourier components $\left\{\mathbf{u}\left(\mathbf{q}, q_{n}\right)\right\}$ can be related to $\mathbf{u}(\mathbf{x}, z)$ through:

$$
\mathbf{u}\left(\mathbf{q}, q_{n}\right)=\frac{1}{L} \int_{0}^{L} d z \int d \mathbf{x} \mathbf{u}(\mathbf{x}, z) \mathrm{e}^{-i\left(\mathbf{q} \cdot \mathbf{x}+q_{n} z\right)}
$$

as can be verified by using equation (9) and the fact that

$$
\int d \mathbf{x} \mathrm{e}^{i\left(\mathbf{q}+\mathbf{q}^{\prime}\right) \cdot \mathbf{x}}=(2 \pi)^{d_{\perp}} \delta\left(\mathbf{q}+\mathbf{q}^{\prime}\right) .
$$


In the above representation of the elastic displacements $\mathbf{u}(\mathbf{x}, z)$, the usual expression of the elastic matrix ${ }^{21,3}$ (here $P_{\alpha \beta}^{L}(\mathbf{q})=q_{\alpha} q_{\beta} / q^{2}$ and $P_{\alpha \beta}^{T}(\mathbf{q})=\delta_{\alpha \beta}-P_{\alpha \beta}^{L}(\mathbf{q})$ are the usual projection operators)

$$
\Phi_{\alpha \beta}\left(\mathbf{q}, q_{n}\right)=\Phi_{L}\left(\mathbf{q}, q_{n}\right) P_{\alpha \beta}^{L}(\mathbf{q})+\Phi_{T}\left(\mathbf{q}, q_{n}\right) P_{\alpha \beta}^{T}(\mathbf{q}),
$$

remains unchanged, except for the appearance of discrete $q_{n}$ modes (instead of a continuous $q_{z}$ variable) and for a slight change in the expression of the longitudinal and transverse components which, due to our specific Fourier transform convention, are now given by :

$$
\begin{aligned}
& \Phi_{L}\left(\mathbf{q}, q_{n}\right)=L\left(c_{11} q^{2}+c_{44} q_{n}^{2}\right), \\
& \Phi_{T}\left(\mathbf{q}, q_{n}\right)=L\left(c_{66} q^{2}+c_{44} q_{n}^{2}\right) .
\end{aligned}
$$

In the above definitions, $c_{11}, c_{66}$ and $c_{44}$ are the compression, shear and tilt moduli of the FLL, respectively. In principle, the compression and tilt moduli have spatial dispersion, i.e. they are wavevector-dependent ${ }^{21}$. Here we shall neglect this dispersion altogether, as it does not affect the long distance behavior of correlation functions.

The elastic Hamiltonian can now be written in the form

$$
H_{e l}=\frac{1}{2} \sum_{n=-\infty}^{\infty} \int_{\mathbf{q}} u_{\alpha}\left(-\mathbf{q},-q_{n}\right) \Phi_{\alpha \beta}\left(\mathbf{q}, q_{n}\right) u_{\beta}\left(\mathbf{q}, q_{n}\right) .
$$

It is to be noted that the elastic matrix $\Phi\left(\mathbf{q}, q_{n}=0\right)$ of the center of mass phonons in three dimensions

$$
\Phi_{\alpha \beta}(\mathbf{q}, 0)=L c_{11} q^{2} P_{\alpha \beta}^{L}(\mathbf{q})+L c_{66} q^{2} P_{\alpha \beta}^{T}(\mathbf{q})
$$

has the same form as the elastic matrix of a two-dimensional lattice of ordinary particles with (two-dimensional) compression and tilt moduli given by $C_{11}=L c_{11}$ and $C_{66}=L c_{66}$ respectively. From the expression (19) of the elastic Hamiltonian, we can easily write for the thermal average $\left\langle u_{\alpha}\left(\mathbf{q}, q_{n}\right) u_{\beta}\left(\mathbf{q}^{\prime}, q_{m}\right)\right\rangle$ the following result (we henceforth use units such that Boltzmann's constant $k_{B}=1$ )

$$
\left\langle u_{\alpha}\left(\mathbf{q}, q_{n}\right) u_{\beta}\left(\mathbf{q}^{\prime}, q_{m}\right)\right\rangle=(2 \pi)^{d_{\perp}} \delta_{n,-m} \delta_{2}\left(\mathbf{q}+\mathbf{q}^{\prime}\right) T G_{\alpha \beta}\left(\mathbf{q}, q_{n}\right),
$$

with the elastic propagator

$$
G_{\alpha \beta}\left(\mathbf{q}, q_{n}\right)=\left[\Phi_{L}\right]^{-1} P_{\alpha \beta}^{L}(\mathbf{q})+\left[\Phi_{T}\right]^{-1} P_{\alpha \beta}^{T}(\mathbf{q}) .
$$

If we call $G_{0, \alpha \beta}(\mathbf{q})$ the elastic propagator of the center of mass mode $G_{\alpha \beta}\left(\mathbf{q}, q_{n}=0\right)$, then it can easily be verified that the mean square relative displacement $C(\mathbf{x})=$ $\left\langle[\mathbf{u}(\mathbf{x})-\mathbf{u}(\mathbf{0})]^{2}\right\rangle$ can be written as the sum

$$
C(\mathbf{x})=C_{0}(\mathbf{x})+C_{1}(\mathbf{x}),
$$

where

$$
C_{0}(\mathbf{x})=2 T \sum_{\alpha} \int_{\mathbf{q}} G_{0, \alpha \alpha}(\mathbf{q})\{1-\cos [\mathbf{q} \cdot \mathbf{x}]\}
$$


is the mean square displacement of the center of mass mode, while

$$
C_{1}(\mathbf{x}, z)=2 T \sum_{\alpha} \sum_{n \neq 0} \int_{\mathbf{q}} G_{\alpha \alpha}\left(\mathbf{q}, q_{n}\right)\left\{1-\cos \left[\mathbf{q} \cdot \mathbf{x}+q_{n} z\right]\right\}
$$

is the corresponding quantity for the internal modes. In what follows, we shall be mainly interested in the center of mass relative displacement $C_{0}(\mathbf{x})$ whose behavior has been largely overlooked in the past, and which will turn out to control the large scale asymptotics of $C(\mathbf{x}, z)$ and the long range translational order in the vortex system.

Using the properties of projection operators, one can easily verify that the trace of the elastic propagator $\mathbf{G}_{0}(\mathbf{q})$ is given by :

$$
\sum_{\alpha} G_{0, \alpha \alpha}(\mathbf{q})=\frac{1}{L}\left(\frac{1}{c_{11} q^{2}}+\frac{1}{c_{66} q^{2}}\right),
$$

and hence we obtain in $2+1$ dimensions :

$$
C_{0}(\mathbf{x}) \simeq \frac{T}{\pi L c_{66}} \int_{0}^{\Lambda} \frac{d q}{q}\left[1-J_{0}(q|\mathbf{x}|)\right]
$$

where we took into account the fact that $c_{66} \ll c_{11}$ (and hence that $c_{11}^{-1}$ is negligibly small compared to $c_{66}^{-1}$ ) for most HTSC. The large distance behavior of the integral on the rhs of the last equation can be obtained in a standard way ${ }^{22}$, with the result :

$$
\int_{0}^{\Lambda} \frac{d q}{q}\left[1-J_{0}(q|\mathbf{x}|)\right]=\ln \Lambda|\mathbf{x}|+\kappa+\mathcal{O}\left((\Lambda|\mathbf{x}|)^{-3 / 2}\right),
$$

where $\kappa=\int_{0}^{1} d u\left(1-J_{0}(u)\right) / u^{2} \simeq-0.116$ is a numerical constant. ${ }^{23}$ Hence, the correlation function $C_{0}(\mathbf{x})$ takes the form ${ }^{24}$

$$
C_{0}(\mathbf{x}) \simeq \frac{T}{\pi L c_{66}}[\ln \Lambda|\mathbf{x}|+\kappa] .
$$

Using the fact that $c_{66}=\varepsilon_{0} / 4 a^{2}$, where $\varepsilon_{0}=\left(\phi_{0} / 4 \pi \lambda\right)^{2}$ (here $\phi_{0}=h c / 2 e$ is the flux quantum ${ }^{25}$ and $\lambda$ is the London penetration depth) and $a$ is the vortex lattice spacing, we obtain

$$
\frac{1}{a^{2}} C_{0}(\mathbf{x}, z) \simeq\left(\frac{T}{T_{0}}\right)[\ln \Lambda|\mathbf{x}|+\kappa]
$$

with the characteristic temperature

$$
T_{0} \simeq \frac{\pi}{4} L \varepsilon_{0} .
$$

The fact that $T_{0}$ is proportional to $L$ is an indication of the fact that the CM mode with compression and shear moduli $C_{11}=L c_{11}$ and $C_{66}=L c_{66}$ respectively, is much stiffer than the internal modes. The characteristic temperature $T_{0}$ of equation (30) is in general a very large temperature for macroscopic sample thicknesses. Using the result ${ }^{3}$

$$
\varepsilon_{0}(K / \AA)=\frac{1.964 \times 10^{8}}{[\lambda(T)(\AA)]^{2}}=\frac{1.964 \times 10^{8}}{[\lambda(0)(\AA)]^{2}}\left(1-\frac{T}{T_{c}}\right)
$$


with $\lambda(0)=1400 \AA$ (typical of HTSC) and $L=1 \mathrm{~cm}$, we find that $T_{0}$ is of the order of $10^{10} \mathrm{~K}$. Even in low temperature superconductors with much higher values of $\lambda(0)$ (typically $\lambda(0)$ is of order $10^{4}$ to $10^{6} \AA$ in these materials), $T_{0}$ is still very high for bulk samples. We therefore conclude that, even though it may appear at first sight from equation (29) that the center of mass positions of the vortices have only quasi long range order, because the prefactor $\left(T / T_{0}\right)$ is extremely small for all temperatures of interest on one hand, and of the very slow variation of the logarithm function on the other, it can be said that the center of mass mode possesses true long range order for all realistic experimental situations.

The situation is, however, much less clear in $1+1$ dimensions (i.e. flux lines confined to a plane). There we find

$$
\begin{aligned}
C_{0}(x) & =\frac{T}{L} \int_{-\Lambda}^{\Lambda} \frac{d q}{2 \pi} \frac{1-\mathrm{e}^{i q x}}{c_{11} q^{2}}, \\
& =\frac{T|x|}{\pi L c_{11}} \int_{0}^{\Lambda|x|} d u \frac{1-\cos u}{u^{2}}, \\
& \simeq \frac{T|x|}{2 L c_{11}},
\end{aligned}
$$

where in going from the first to the second line we used the change of variables $q=\Lambda|\mathbf{x}|$, and where we obtained the last line by sending $\Lambda|\mathbf{x}|$ to infinity and using the result ${ }^{26} \int_{0}^{\infty} d u(1-\cos u) / u^{2}=\pi / 2$. Here again, the survival of positional order in a given sample of thickness $L$ will depend on the numerical value of $\left(T / \pi L c_{11}\right)$. If the vortices are so far apart from each other that the condition $a>\lambda$ is satisfied, then one can use the following expression for the compression modulus ${ }^{27,3}$

$$
c_{11}=\frac{\pi^{2} T^{2} n^{4}}{c_{44}} \frac{1}{(1-D n)^{2}},
$$

where $n=1 / a$ is the density of flux lines, and $D \approx \lambda$ is the range of the interaction between vortices. Assuming that the tilt modulus $c_{44}$ is of order $n \varepsilon_{0}$, and neglecting the factor $(1-D n)^{-2}$ which is of order unity for $a>\lambda$, we obtain ${ }^{4}$

$$
c_{11} \simeq \frac{\pi^{2} T^{2}}{\varepsilon_{0} a^{3}} .
$$

Using this expression in equation (32), we are led to the following numerical estimate for the displacement correlation function:

$$
\frac{1}{a^{2}} C_{0}(x)=\frac{1.964 \times 10^{8}(\mathrm{~K} \cdot \AA)}{2 \pi^{2} T L}\left(\frac{a}{\lambda(T)}\right)^{2} \frac{|x|}{a},
$$

where the expression (31) of $\varepsilon_{0}$ has been used. Thus we see that, even at very low temperatures (of order, say, a few kelvins) and large sample thicknesses $(L \sim 1 \mathrm{~cm}=$ $10^{8} \AA$ ), long range order will be destroyed on a relatively short distance, of order a few lattice constants $a$. This is an important result in view of the fact that it is a generally accepted fact that line lattices in $1+1$ dimensions have quasi-long range translational order ${ }^{3}$. Here we have just shown that there might be physical 
regimes where the very notion of an ordered lattice in the $1+1$ geometry becomes questionable, even in the absence of disorder.

For higher vortex densities, no closed form for the elastic constants $c_{11}$ and $c_{44}$ in a film of finite thickness, satisfying the correct boundary conditions for magnetic fields and supercurrents at the surface of the film ${ }^{28}$, seems to exist in the literature. This case obviously deserves further attention but will not be considered in any detail here. It is however clear that, if $c_{11}$ becomes of order $n \varepsilon_{0}$ when $a \ll \lambda$, then the center of mass correlation function will behave like $\sim\left(T / T_{0}\right)|x| a$, with $T_{0}$ given by equation (30), and long range order will be effectively recovered again at all temperatures and realistic sample sizes.

\section{Positional order in the presence of a random pinning potential}

We now turn our attention to the more interesting problem of the positional order of the FLL in the presence of disorder. To start with, we shall first apply Larkin's argument to the center of mass mode of the flux lines, before presenting a more careful replica analysis in Section 3.2.

\subsection{Larkin's argument applied to the center of mass mode}

Following Larkin's original work ${ }^{5,17}$, we assume that the action of the random impurities on the FLL can be represented by a random pinning force $\mathbf{F}(\mathbf{r})$ whose

probability distribution is assumed to be Gaussian with zero mean and correlations (we remind the reader that the overbar denotes an average over the disorder)

$$
\overline{F_{\alpha}(\mathbf{x}, z) F_{\beta}\left(\mathbf{x}^{\prime}, z^{\prime}\right)}=W \delta_{\alpha \beta} \delta\left(\mathbf{x}-\mathbf{x}^{\prime}\right) \delta\left(z-z^{\prime}\right),
$$

or, in Fourier space,

$$
\overline{F_{\alpha}\left(\mathbf{q}, q_{n}\right) F_{\beta}\left(\mathbf{q}^{\prime}, q_{m}\right)}=L W \delta_{\alpha \beta}(2 \pi)^{2} \delta\left(\mathbf{q}+\mathbf{q}^{\prime}\right) \delta_{n,-m} .
$$

The Hamiltonian of the flux line system is now given by:

$$
H=H_{e l}-\int_{0}^{L} d z \int d \mathbf{x} \mathbf{F}(\mathbf{x}, z) \cdot \mathbf{u}(\mathbf{x}, z),
$$

where $H_{e l}$ is the elastic Hamiltonian of equation (19). Note that, as it stands, the random pinning force $\mathbf{F}(\mathbf{x}, z)$ only depends on the "ideal" positions of flux lines "x" and not on their displacements $\mathbf{u}(\mathbf{x}, z)$. As we will see below (in paragraph 3.2), it is more appropriate to represent the random impurities by a random pinning potential $V(\mathbf{x}+\mathbf{u}(\mathbf{x}, z))$, and the random force term in equation (38) can be thought of as the leading term in a Taylor expansion of the random potential in terms of the phonon field $\{\mathbf{u}(\mathbf{x}, z)\}$.

At low temperatures, the statistical mechanics associated with the Gibbs measure $\exp (-H / T)$ is dominated by those configurations of the displacement field $\{\mathbf{u}(\mathbf{x}, z)\}$ for which $H$ is minimal ${ }^{17,29,30}$. Minimization $^{31}$ of $H$ with respect to $\left\{\mathbf{u}\left(\mathbf{q}, q_{n}\right)\right\}$ leads to the result

$$
u_{\alpha}\left(\mathbf{q}, q_{n}\right)=G_{\alpha \beta}\left(\mathbf{q}, q_{n}\right) F_{\beta}\left(\mathbf{q}, q_{n}\right),
$$


where $G_{\alpha \beta}\left(\mathbf{q}, q_{n}\right)$ is the elastic ptopagator of equation (22). It then follows that the correlation function $\left\langle u_{\alpha}(\mathbf{r}) u_{\beta}\left(\mathbf{r}^{\prime}\right)\right\rangle$ averaged over the configurations of the random force (36) is given by:

$$
\begin{aligned}
\overline{\left\langle u_{\alpha}(\mathbf{r}) u_{\beta}\left(\mathbf{r}^{\prime}\right)\right\rangle} & =\sum_{n, m} \int_{\mathbf{q}} \int_{\mathbf{q}^{\prime}} G_{\alpha \gamma}\left(\mathbf{q}, q_{n}\right) G_{\beta \delta}\left(\mathbf{q}^{\prime}, q_{m}\right) \times \\
& \times \overline{F_{\alpha}\left(\mathbf{q}, q_{n}\right) F_{\beta}\left(\mathbf{q}^{\prime}, q_{m}\right)} \mathrm{e}^{i\left(\mathbf{q} \cdot \mathbf{x}+q_{n} z\right)} \mathrm{e}^{i\left(\mathbf{q} \cdot \mathbf{x}^{\prime}+q_{m} z^{\prime}\right)} .
\end{aligned}
$$

Using expression (36) of the random force correlator, we obtain the following result

$$
\left\langle u_{\alpha}(\mathbf{r}) u_{\beta}\left(\mathbf{r}^{\prime}\right)\right\rangle=W L \sum_{n} \int_{\mathbf{q}} G_{\alpha \gamma}\left(\mathbf{q}, q_{n}\right) G_{\beta \gamma}\left(-\mathbf{q},-q_{n}\right) \mathrm{e}^{i \mathbf{q} \cdot\left(\mathbf{x}-\mathbf{x}^{\prime}\right)} \mathrm{e}^{i q_{n}\left(z-z^{\prime}\right)},
$$

which implies that the relative displacement correlator $C(\mathbf{x}, z)=\langle[\mathbf{u}(\mathbf{x}, z)-$ $\left.\mathbf{u}(\mathbf{0}, 0)]^{2}\right\rangle$ is given by

$$
C(\mathbf{x}, z)=2 W L \sum_{n} \int_{\mathbf{q}} G_{\alpha \beta}\left(\mathbf{q}, q_{n}\right) G_{\alpha \beta}\left(-\mathbf{q},-q_{n}\right)\left[1-\mathrm{e}^{i\left(\mathbf{q} \cdot \mathbf{x}+q_{n} z\right)}\right] .
$$

Now if we use expression (22) of the elastic propagator $G\left(\mathbf{q}, q_{n}\right)$, we find that the usual expression ${ }^{5,29,3}$ of the correlator $C(\mathbf{x}, z)$ in $d$ dimensions

$$
C(\mathbf{x}, z) \simeq 2 W \int \frac{d^{d} \mathbf{k}}{(2 \pi)^{d}} \frac{1-\cos \left(\mathbf{k}_{\perp} \cdot \mathbf{x}+k_{z} z\right)}{\left[c_{66} k_{\perp}^{2}+c_{44}(k) k_{z}^{2}\right]^{2}}
$$

is replaced by (we remind the reader that $d_{\perp}=d-1$ )

$$
C(\mathbf{x}, z) \simeq \frac{2 W}{L} \sum_{n=-\infty}^{\infty} \int \frac{d^{d_{\perp}} \mathbf{q}}{(2 \pi)^{d_{\perp}}} \frac{1-\cos \left(\mathbf{q} \cdot \mathbf{x}+q_{n} z\right)}{\left[c_{66} q^{2}+c_{44} q_{n}^{2}\right]^{2}} .
$$

Obviously, if we transform the summation in this last expression into an integral we recover the $d$-dimensional result (43), with the long distance behavior $C(\mathbf{r}) \sim r^{4-d}$. However, here we are mainly interested in the fluctuations of the CM mode which, we would like to argue, are the most relevant ones for positional order in samples of finite thickness (more specifically, in samples whose thickness $L$ along the direction of flux lines is much smaller than their transverse size $L_{\perp}$ ). We shall therefore write

$$
C(\mathbf{x}, z)=C_{0}(\mathbf{x})+C_{1}(\mathbf{x}, z),
$$

where

$$
C_{0}(\mathbf{x})=\frac{2 W}{L} \int \frac{d^{d_{\perp}} \mathbf{q}}{(2 \pi)^{d_{\perp}}} \frac{1-\cos (\mathbf{q} \cdot \mathbf{x})}{c_{66}^{2} q^{4}}
$$

is the correlator of the center of mass positions, while

$$
C_{1}(\mathbf{x}, z)=\frac{2 W}{L} \sum_{n \neq 0} \int \frac{d^{d_{\perp}} \mathbf{q}}{(2 \pi)^{d_{\perp}}} \frac{1-\cos \left(\mathbf{q} \cdot \mathbf{x}+q_{n} z\right)}{\left[c_{66} q^{2}+c_{44} q_{n}^{2}\right]^{2}}
$$

corresponds to the internal modes of the FLL. Taking $d_{\perp}=2$ and transforming the above sum into an integral, we obtain the usual large distance result in $d=3$ 
dimensions ${ }^{5,29,3}$ (recall that here, we neglect the dispersion of the elastic constants; taking the dispersion of $c_{44}$ into account leads to a different behavior at short distances):

$$
C_{1}(\mathbf{x}, z) \simeq \frac{W \lambda}{2 \pi^{2} c_{66}^{3 / 2} c_{44}^{1 / 2}}\left(\frac{|\mathbf{x}|^{2}}{\lambda^{2}}+\frac{a^{2} z^{2}}{\lambda^{4}}\right)^{1 / 2} .
$$

On the other hand, we have for the center of mass positions :

$$
\begin{aligned}
C_{0}(\mathbf{x}) & =\frac{W}{\pi L c_{66}^{2}} \int_{1 / L_{\perp}}^{\Lambda} d q \frac{1-J_{0}(q|\mathbf{x}|)}{q^{3}} \\
& =\frac{W|\mathbf{x}|^{2}}{\pi L c_{66}^{2}} \int_{|\mathbf{x}| / L_{\perp}}^{\Lambda|\mathbf{x}|} d u \frac{1-J_{0}(u)}{u^{3}}
\end{aligned}
$$

where, in going from the first to the second line we made the change of variables $u=q x$, and where we used the inverse of the transverse size of the system, $1 / L_{\perp}$, as an infrared cut-off to insure the convergence of the integral as $q \rightarrow 0$. At large distances, the upper bound of the integral on the rhs of equation (48) can be replaced by infinity, with the result (we assume that $|\mathbf{x}| \ll L_{\perp}$ ):

$$
\int_{|\mathbf{x}| / L_{\perp}}^{\Lambda|\mathbf{x}|} d u \frac{1-J_{0}(u)}{u^{3}} \simeq \int_{|\mathbf{x}| / L_{\perp}}^{1} d u \frac{1-J_{0}(u)}{u^{3}}+\int_{1}^{\infty} d u \frac{1-J_{0}(u)}{u^{3}}
$$

Now, on the interval $\left[x / L_{\perp}, 1\right]$, we can approximate $J_{0}(u)=1-u^{2} / 4+\mathrm{o}\left(u^{4}\right)$. This leads to

$$
\int_{x / L_{\perp}}^{1} d u \frac{1-J_{0}(u)}{u^{3}} \approx \frac{1}{4} \ln \left(L_{\perp} / x\right) .
$$

On the other hand, $\int_{1}^{\infty} d u \frac{1-J_{0}(u)}{u^{3}}$ is just a numerical constant, whose value we shall denote by $\alpha$, and which is approximately given by $\alpha \simeq 0.287$, so that equation (48) finally yields :

$$
C_{0}(\mathbf{x}) \simeq \frac{W|\mathbf{x}|^{2}}{4 \pi L c_{66}^{2}}\left[\ln \left(L_{\perp} / x\right)+\alpha^{\prime}\right],
$$

where we defined $\alpha^{\prime}=4 \alpha \simeq 1.15$. Apart from the unimportant numerical constant $\alpha^{\prime}$ (which can be absorbed in the cut-off $R$ ), this result is very similar to what has been obtained a long time ago ${ }^{29,32}$ for thin superconducting films. Here we see that this result in fact holds true for the CM mode of the flux lines in samples of arbitrary thickness.

The above perturbative analysis is correct up to the length scale $x_{c}$ such that $C_{0}\left(x_{c}\right) \simeq \xi^{2}$. The quantity $x_{c}$ defines a length at which the center of mass mode of vortices has "random-walked" a distance $\xi$ from a given initial position. Using equation (51), we obtain that $x_{c}$ is the solution of the following equation (henceforth we omit the constant $\alpha$ from our considerations)

$$
x_{c}^{2} \simeq \frac{4 \pi L c_{66}^{2} \xi^{2}}{W \ln \left(L_{\perp} / x_{c}\right)} .
$$


A good approximation to this last quantity is obtained by replacing $x_{c}$ inside the argument of the logarithm by $x_{0}=\left(4 \pi L c_{66}^{2} \xi^{2} / W\right)^{1 / 2}$. We then have, to logarithmic accuracy:

$$
x_{c} \simeq\left[\frac{4 \pi L c_{66}^{2}}{W \ln \left(L_{\perp} / x_{0}\right)}\right]^{1 / 2} \xi .
$$

The important thing to note about $x_{c}$ is that it varies with the sample thickness $L$ as $L^{1 / 2}$, and hence is very large for bulk samples. Let us for example compare $x_{c}$ to the in-plane characteristic length $R_{c}^{\perp}$ for the internal modes. For the sake of argument, we define $R_{c}^{\perp}$ as the length scale at which the equal height correlator $C_{1}(\mathbf{x}, 0)=W|\mathbf{x}| /\left(2 \pi^{2} c_{66}^{3 / 2} c_{44}^{1 / 2}\right)$ reaches the value $\xi^{2}$. We have

$$
R_{c}^{\perp}=\frac{2 \pi^{2}}{W} c_{66}^{3 / 2} c_{44}^{1 / 2} \xi^{2}
$$

and hence

$$
\begin{aligned}
\frac{x_{c}}{R_{c}^{\perp}} & =\frac{(L W)^{1 / 2}}{\pi^{3 / 2} \xi c_{66}^{1 / 2} c_{44}^{1 / 2} \sqrt{\ln \left(L_{\perp} / x_{0}\right)}}, \\
& =\frac{(L W)^{1 / 2} a^{3}}{\pi^{2} \xi \lambda \varepsilon_{0} \sqrt{\ln \left(L_{\perp} / x_{0}\right)}},
\end{aligned}
$$

where, in going from the first to the second line, we used the fact that $c_{44}=B^{2} / 4 \pi$ (with $B=\phi_{0} / a^{2}$ the magnetic induction inside the superconductor) and hence that $^{3}$

$$
\sqrt{c_{66} c_{44}}=\frac{\pi \lambda \varepsilon_{0}}{a^{3}} .
$$

Now, if we use $W \approx \varepsilon_{0}^{2} / d_{0}^{3}$, where we denote by $d_{0}$ the average distance between impurities in the superconducting sample, we obtain

$$
\frac{x_{c}}{R_{c}^{\perp}} \approx \frac{\kappa}{\pi^{2} \sqrt{\ln \left(L_{\perp} / x_{0}\right)}}\left(\frac{a}{\lambda}\right)^{2}\left(\frac{a}{d_{0}}\right) \sqrt{\frac{L}{d_{0}}},
$$

with $\kappa=\lambda / \xi$. Using $\kappa=100, \lambda(0)=1400 \AA, a=500 \AA, d=100 \AA$ and $L_{\perp}=L=$ $1 \mathrm{~cm}$ we obtain

$$
\frac{x_{c}}{R_{c}^{\perp}} \approx 6 \times 10^{2}\left(1-T / T_{c}\right) .
$$

This shows that, now matter how large the internal fluctuations of the flux lines are, the center of mass mode of the flux lines experiences much smaller fluctuations and has a much larger Larkin length than the internal modes.

It is interesting to see how the above results are modified if correlated disorder is considered instead of point disorder. In that case, the random force correlations are given by:

$$
\overline{F_{\alpha}(\mathbf{x}) F_{\beta}\left(\mathbf{x}^{\prime}\right)}=W_{c} \delta_{\alpha \beta} \delta\left(\mathbf{x}-\mathbf{x}^{\prime}\right),
$$

with $^{33} W_{c} \approx \varepsilon_{0}^{2} /\left(a^{2} d_{0}^{2}\right)$. An immediate consequence of the fact that the random force does not depend on $z$ is that $F_{\alpha}(\mathbf{x})$ will couple only to the center of mass 
mode of the flux lines, as can be easily seen using the fact that $\int_{0}^{L} d z \mathbf{u}_{1}(z)=0$ in the last term of equation (38) (where we now let $\mathbf{F}(\mathbf{x}, z)=\mathbf{F}(\mathbf{x})$ ). The Hamiltonian of the flux line system can now be written in the form (here and in what follows we concentrate on $d=3$ dimensions) :

$$
\begin{aligned}
H & =\frac{1}{2} \sum_{n \neq 0} \int_{\mathbf{q}} u_{\alpha}\left(-\mathbf{q},-q_{n}\right) \Phi_{\alpha \beta}\left(\mathbf{q}, q_{n}\right) u_{\beta}\left(\mathbf{q}, q_{n}\right)+ \\
& +\frac{1}{2} \sum_{n \neq 0} \int_{\mathbf{q}} u_{0, \alpha}(-\mathbf{q}) \Phi_{0, \alpha \beta}(\mathbf{q}) u_{0, \beta}(\mathbf{q})-L \int d \mathbf{x} \mathbf{F}(\mathbf{x}) \cdot \mathbf{u}_{0}(\mathbf{x}),
\end{aligned}
$$

from which we obtain the interesting result (within the Larkin approximation of ignoring the $\mathbf{u}$ dependence of the random pinning force $\mathbf{F}$ ) that correlation functions for the internal modes fluctuations remain unchanged and are in fact the same as their pure counterparts. Only the CM mode is affected by correlated disorder in first order perturbation theory, and the CM part of the Hamiltonian (60) maps onto the Hamiltonian of a two-dimensional system with compression and bulk moduli given by $C_{11}=L c_{11}$ and $C_{66}=L c_{66}$ respectively, subject to the random force $\tilde{\mathbf{F}}(\mathbf{x})=$

$L \mathbf{F}(\mathbf{x})$ whose variance is now given by $\tilde{W}_{c}=L^{2} W_{c}$. The relative displacement of the center of mass mode $C_{0}(\mathbf{x})$ is then obtained in a very natural way as

$$
\begin{aligned}
C_{0}(\mathbf{x}) & =2 \tilde{W}_{c} \int \frac{d^{2} \mathbf{q}}{(2 \pi)^{2}} G_{\alpha \beta}(\mathbf{q}, 0) G_{\alpha \beta}(-\mathbf{q}, 0)\left[1-\mathrm{e}^{i \mathbf{q} \cdot \mathbf{x}}\right], \\
& =\frac{2 W_{c}}{c_{66}^{2}} \int \frac{d^{2} \mathbf{q}}{(2 \pi)^{2}} \frac{1-\cos (\mathbf{q} \cdot \mathbf{x})}{q^{4}} .
\end{aligned}
$$

A calculation similar to the one carried out in equations (49)-(51) leads to the result

$$
C_{0}(\mathbf{x})=\frac{W_{c}|\mathbf{x}|^{2}}{4 \pi c_{66}^{2}} \ln \left(L_{\perp} / x\right)
$$

which is the result obtained by Nelson and Vinokur ${ }^{33}$. Unlike the displacement correlator in the presence of point disorder, equation (51), the correlator (62) does not depend on the sample thickness $L$. The resulting size $x_{c}$ of the perturbative region for the $\mathrm{CM}$ mode, which verifies the implicit equation

$$
x_{c}=\frac{4 \pi c_{66}^{2} \xi^{2}}{W_{c} \ln \left(L_{\perp} / x_{c}\right)},
$$

does not increase with $L$, which translates the fact that columnar pins are much more effective in disrupting the long range order of the FLL than point-like pinners.

\subsection{Variational analysis}

As is well known, the Larkin analysis of the previous section breaks down beyond the Larkin length $x_{c}$. To find the long distance behavior of correlation functions, one has to resort to a more careful kind of analysis. Here we shall use the Gaussian variational method ${ }^{14,11,13,20}(\mathrm{GVM})$ to find the long distance behavior of the 
center of mass relative displacement $C_{0}(\mathbf{x})$. The GVM has been used in the past to study the effect of disorder on elastic manifolds ${ }^{14}$ and flux lattices ${ }^{11,13,20}$, and although uncontrolled (due to the absence of a small expansion parameter), it has been shown ${ }^{13,15}$ to yield results that are in qualitative agreement with functional renormalization group calculations.

Here, we shall restrict ourselves to the three dimensional case and to isotropic elasticity, the generalization to the more realistic case of different elastic moduli being relatively straightforward. Following ref. ${ }^{13}$, in this subsection we shall use the following Hamiltonian

$$
H=\int d \mathbf{x} d z \frac{1}{2} c\left[\left(\partial_{z} \mathbf{u}\right)^{2}+\left(\nabla_{\mathbf{x}} \cdot \mathbf{u}\right)^{2}\right]+\int d \mathbf{x} d z V(\mathbf{x}, z) \rho(\mathbf{x}, z),
$$

where the vortex density is given by

$$
\rho(\mathbf{x}, z)=\sum_{i} \delta_{2}\left(\mathbf{x}-\mathbf{x}_{i}(z)\right)=\sum_{i} \delta_{2}\left(\mathbf{x}-\mathbf{R}_{i}-\mathbf{u}_{i}(z)\right),
$$

and where $V(\mathbf{x}, z)$ is a Gaussian random potential with zero mean and correlations

$$
\overline{V(\mathbf{x}, z) V\left(\mathbf{x}^{\prime}, z^{\prime}\right)}=\Delta\left(\mathbf{x}-\mathbf{x}^{\prime}, z-z^{\prime}\right) .
$$

Using the replica "trick"

$$
\overline{\ln Z}=\lim _{n \rightarrow 0} \frac{\overline{Z^{n}}-1}{n}
$$

to average the free energy over disorder, we obtain the following effective Hamiltonian

$$
\begin{aligned}
H_{e f f} & =\frac{1}{2} \sum_{a=1}^{n} \int d \mathbf{x} d z c\left[\left(\partial_{z} \mathbf{u}_{a}\right)^{2}+\left(\nabla_{\mathbf{x}} \mathbf{u}_{a}\right)^{2}\right]+ \\
& -\frac{1}{2 T} \sum_{a, b} \int d \mathbf{x} d z \int d \mathbf{x}^{\prime} d z^{\prime} \rho_{a}(\mathbf{x}, z) \rho_{b}\left(\mathbf{x}^{\prime}, z^{\prime}\right) \Delta\left(\mathbf{x}-\mathbf{x}^{\prime}, z-z^{\prime}\right) .
\end{aligned}
$$

Now, using the following decomposition of the density ${ }^{34,35,13}$

$$
\rho(\mathbf{x}, z) \simeq \rho_{0}\left[1-\partial_{\alpha} u_{\alpha}(\mathbf{x}, z)\right]+\rho_{0} \sum_{\mathbf{K} \neq 0} \rho_{\mathbf{K}}(\mathbf{x}) \mathrm{e}^{i \mathbf{K} \cdot \mathbf{x}},
$$

where $\rho_{\mathbf{K}}(\mathbf{x})=\mathrm{e}^{-i \mathbf{K} \cdot \mathbf{u}(\mathbf{x}, z)}$, and discarding rapidly oscillating terms, we obtain from equation (68) :

$$
\begin{aligned}
H_{e f f} & =\frac{1}{2} \sum_{a=1}^{n} \int d \mathbf{x} d z c\left[\left(\partial_{z} \mathbf{u}_{a}\right)^{2}+\left(\nabla_{\mathbf{x}} \mathbf{u}_{a}\right)^{2}\right]-\sum_{a, b} \int d \mathbf{x} d z\left\{\frac{\Delta_{0}}{2 T} \partial_{\alpha} u_{\alpha}^{a}(\mathbf{x}, z) \partial_{\beta} u_{\beta}^{b}(\mathbf{x}, z)\right. \\
& \left.+\sum_{\mathbf{K} \neq 0} \frac{\Delta_{\mathbf{K}}}{2 T} \cos \left(\mathbf{K} \cdot\left(\mathbf{u}_{a}(\mathbf{x}, z)-\mathbf{u}_{b}(\mathbf{x}, z)\right)\right)\right\},
\end{aligned}
$$


where we defined

$$
\begin{aligned}
\Delta_{0} & =\rho_{0}^{2} \int d \mathbf{x} \Delta(\mathbf{x}), \\
\Delta_{\mathbf{K}} & =\rho_{0}^{2} \int d \mathbf{x} \Delta(\mathbf{x}) \mathrm{e}^{i \mathbf{K} \cdot \mathbf{x}} .
\end{aligned}
$$

The highly nonlinear form of $H_{\text {eff }}$ precludes exact analysis. In order to make progress a common way is to use the so-called Gaussian variational method (GVM), which has been developed by Mézard and Parisi ${ }^{14}$ in the context of random manifolds, and was first applied to the vortex lattice by Bouchaud, Mézard and Yedidia ${ }^{11}$. This method consists in trying to find the best quadratic variational Hamiltonian $H_{v}$ to describe the full nonlinear problem using Boguliubov's variational free energy ${ }^{36,22}$

$$
F_{v}=\left\langle H-H_{v}\right\rangle_{v}-T \ln Z_{v},
$$

where $\langle\cdots\rangle_{v}$ denotes averaging with statistical weight $\exp \left(-H_{v} / T\right) / Z_{v}$, and where $Z_{v}=\operatorname{Tr}\left(\mathrm{e}^{-H_{v} / T}\right)$. As a trial Hamiltonian we take

$$
H_{v}=\frac{1}{2} \sum_{n} \int \frac{d^{d} \mathbf{q}}{(2 \pi)^{d}}\left(G^{-1}\right)_{a b}\left(\mathbf{q}, q_{n}\right) \mathbf{u}_{a}\left(\mathbf{q}, q_{n}\right) \cdot \mathbf{u}_{b}\left(-\mathbf{q},-q_{n}\right)
$$

where $\left(G^{-1}\right)_{a b}$ is an $n \times n$ matrix of variational parameters. The variational free energy is then given by

$$
\begin{aligned}
F_{v} & =\frac{1}{2} \sum_{a, b} \sum_{m} \int_{\mathbf{q}}\left\{\left[c\left(q^{2}+q_{m}^{2}\right) \delta_{a b}-\frac{\Delta_{0}}{T} q^{2}\right] G_{a b}\left(\mathbf{q}, q_{m}\right)-d_{\perp} T[\ln (T G)]_{a a} \delta_{a b}\right\} \\
& -\sum_{a, b} \sum_{\mathbf{K} \neq 0} \frac{\Delta_{K}}{2 T} \mathrm{e}^{-\frac{1}{2} K^{2} C_{a b}(\mathbf{x}=0, z=0)}
\end{aligned}
$$

where we defined the difference correlation function (note that in this section, difference correlation functions $C(\mathbf{x}, z)$ are defined with an extra factor $1 / d_{\perp}$, and that throughout the rest of this paper, no summation is implied on repeated indices) :

$$
\begin{aligned}
C_{a b}(\mathbf{x}, z) & =\frac{1}{d_{\perp}}\left\langle\left[\mathbf{u}_{a}(\mathbf{x}, z)-\mathbf{u}_{b}(\mathbf{0}, 0)\right]^{2}\right\rangle \\
& =T \sum_{n} \int_{\mathbf{q}}\left[G_{a a}\left(\mathbf{q}, q_{n}\right)+G_{b b}\left(\mathbf{q}, q_{n}\right)-2 \cos \left(\mathbf{q} \cdot \mathbf{x}+q_{n} z\right) G_{a b}\left(\mathbf{q}, q_{n}\right)\right](.76)
\end{aligned}
$$

Minimization of the variational free energy (75) with respect to $\left[G\left(\mathbf{q}, q_{n}\right)\right]_{a b}$ for $a \neq b$ leads to the following expression ${ }^{13}$

$$
\begin{aligned}
{\left[G^{-1}\left(\mathbf{q}, q_{n}\right)\right]_{a b} } & =c\left(q^{2}+q_{n}^{2}\right) \delta_{a b}-\frac{\Delta_{0}}{d_{\perp} T} q^{2} \\
& -\sum_{K \neq 0} \frac{K^{2} \Delta_{\mathbf{K}}}{d_{\perp} T} \exp \left(-\frac{1}{2} K^{2} C_{a b}(\mathbf{x}=0, z=0)\right) .
\end{aligned}
$$

Defining the self energy matrix $\sigma_{a b}$ by

$$
\left[G^{-1}\left(\mathbf{q}, q_{n}\right)\right]_{a b}=c\left(q^{2}+q_{n}^{2}\right) \delta_{a b}-\sigma_{a b},
$$


we obtain

$$
\sigma_{a \neq b}=+\frac{\Delta_{0}}{d_{\perp} T} q^{2}+\sum_{K \neq 0} \frac{K^{2} \Delta_{\mathbf{K}}}{d_{\perp} T} \exp \left(-\frac{1}{2} K^{2} C_{a b}(\mathbf{x}=0, z=0)\right),
$$

while for $a=b$, we obtain

$$
\sigma_{a a}=-\sum_{b \neq a} \sigma_{a b} .
$$

Two types of solution can exist for the variational equations (79)-(80). The first type is the so-called "replica-symmetric" solution which preserves the permutation symmetry between replicas, while the second type is a "replica-broken" solution in which permutation symmetry between replicas is violated. Here we shall not give much details on the replica symmetric solution, and refer the reader to reference ${ }^{13}$ where more information can be found. The only change with respect to the latter reference is in the form of the diagonal correlation function $G_{a a}\left(\mathbf{q}, q_{n}\right)$ which, because the $q_{z}$ modes in our description are discrete, now takes the form

$$
G_{a a}\left(\mathbf{q}, q_{n}\right)=\frac{1}{c\left(q^{2}+q_{n}^{2}\right)}+\frac{1}{c^{2}\left(q^{2}+q_{n}^{2}\right)^{2} d_{\perp} T} \sum_{\mathbf{K}} K^{2} \Delta_{\mathbf{K}} \mathrm{e}^{-K^{2} \ell^{2} / 2} .
$$

In the above expression, $\ell$ is the (thermal) Lindemann length ${ }^{13}$

$$
\begin{aligned}
\ell^{2} & =\frac{2 T}{L} \sum_{n=-\infty}^{\infty} \int_{0}^{\Lambda} \frac{d^{d-1} \mathbf{q}}{(2 \pi)^{d-1}} \frac{1}{c\left(q^{2}+q_{n}^{2}\right)}, \\
& \simeq \frac{T}{\pi c a},
\end{aligned}
$$

where the second line refers to $d=2+1$ dimensions. Due to the factor $1 /\left(q^{2}+q_{n}^{2}\right)^{2}$ on the rhs of equation (81), we find that the center of mass relative displacements grow as

$$
C_{0, a a}(\mathbf{x}) \sim x^{5-d},
$$

which, for $d=3$ is nothing but the Larkin result of eq. (51).

The analysis of quadratic perturbations, in replica space, about the replicasymmetric solution described above is most conveniently done using the eigenvalue of the so-called "replicon" mode ${ }^{37,14}$, and such an analysis (see Appendix Appendix A) shows that, for the particular case $d=3$, there exists a temperature $T_{c}=4 \pi c L / K_{0}^{2}$ between a high-temperature, replica symmetric phase, and a low temperature glassy phase where replica symmetry is broken. This transition temperature is exactly the same as the one obtained previously in the context of correlated-disorder ${ }^{20}$. Of course, for macroscopic samples $(L \gg a)$ this transition temperature $T_{c}$ is very large and is certainly not experimentally accessible, but from a conceptual point of view it is important to realize its existence for three dimensional flux lattices in the presence of point disorder.

Now, in the low temperature, replica broken phase, the $n \rightarrow 0$ limit of matrices in replica space become functions ${ }^{38,14}$ of a real variable $v$ which parametrizes pairs of 
low lying metastable states. ${ }^{39}$ Following ${ }^{14,13}$ we let $\tilde{G}\left(\mathbf{q}, q_{n}\right)=G_{a a}\left(\mathbf{q}, q_{n}\right), \tilde{C}(\mathbf{x}, z)=$ $C_{a a}(\mathbf{x}, z)$, and parametrize $G_{a b}\left(\mathbf{q}, q_{n}\right)$ and $C_{a b}(\mathbf{x}, z)$ for $a \neq b$ by $G\left(\mathbf{q}, q_{n} ; v\right)$ and $C(\mathbf{x}, z ; v)$ respectively, with $0<v<1$. The saddle point equation (79) can then be rewritten in the form :

$$
\sigma(v)=\sum_{\mathbf{K}} \frac{\Delta_{\mathbf{K}}}{d_{\perp} T} K^{2} \mathrm{e}^{-\frac{1}{2} K^{2} C(0, v)},
$$

where we neglected the subdominant ${ }^{13} K=0$ component of the disorder (the $\Delta_{0} q^{2} / d_{\perp} T$ term) and where

$$
C(\mathbf{0}, 0 ; v)=\frac{2 T}{L} \sum_{n=-\infty}^{\infty} \int \frac{d^{d-1} \mathbf{q}}{(2 \pi)^{d-1}}\left(\tilde{G}\left(\mathbf{q}, q_{n}\right)-G\left(\mathbf{q}, q_{n} ; v\right)\right) .
$$

The algebraic rules for inversion of hierarchical matrices ${ }^{14,13}$ give us :

$$
C(\mathbf{0}, 0 ; v)=C\left(\mathbf{0}, 0 ; v_{c}\right)+\frac{1}{L} \sum_{n=-\infty}^{\infty} \int_{v}^{v_{c}} d u \int \frac{d^{d-1} \mathbf{q}}{(2 \pi)^{d-1}} \frac{2 T \sigma^{\prime}(u)}{\left(c\left(q^{2}+q_{n}^{2}\right)+[\sigma](u)\right)^{2}}
$$

where we defined $[\sigma](v)=v \sigma(v)-\int_{0}^{v} d u \sigma(u)$ and

$$
C\left(\mathbf{0}, 0 ; v_{c}\right)=\frac{1}{L} \sum_{n=-\infty}^{\infty} \int \frac{d^{d-1} \mathbf{q}}{(2 \pi)^{d-1}} \frac{2 T}{c\left(q^{2}+q_{n}^{2}\right)+[\sigma]\left(v_{c}\right)}
$$

Taking the derivative of equation (84) with respect to $v$ (keeping only ${ }^{13}$ the reciprocal lattice vectors $\mathbf{K}$ such that $K=K_{0}$ ), we obtain

$$
\begin{aligned}
1 & =\sigma(v) \cdot \frac{1}{L} \sum_{n=-\infty}^{\infty} \int \frac{d^{d-1} \mathbf{q}}{(2 \pi)^{d-1}} \frac{T K_{0}^{2}}{\left(c\left(q^{2}+q_{n}^{2}\right)+[\sigma](v)\right)^{2}}, \\
& =\frac{\sigma(v)}{L} \int \frac{d^{d-1} \mathbf{q}}{(2 \pi)^{d-1}} \frac{T K_{0}^{2}}{\left(c q^{2}+[\sigma](v)\right)^{2}}+\frac{\sigma(v)}{L} \sum_{n \neq 0} \int \frac{d^{d-1} \mathbf{q}}{(2 \pi)^{d-1}} \frac{T K_{0}^{2}}{\left.\left(c\left(q^{2}+q_{n}^{2}\right)+[\sigma](v)\right)^{2}\right)}
\end{aligned}
$$

Transforming the sum on the rhs into an integral, we obtain

$$
\begin{aligned}
1 & \simeq \frac{\sigma(v)}{L} \int \frac{d^{d-1} \mathbf{q}}{(2 \pi)^{d-1}} \frac{T K_{0}^{2}}{\left(c q^{2}+[\sigma](v)\right)^{2}}+\sigma(v) \int \frac{d^{d} \mathbf{k}}{(2 \pi)^{d}} \frac{T K_{0}^{2}}{\left(c k^{2}+[\sigma](v)\right)^{2}} \\
& =\frac{\sigma(v)}{L}\left(\frac{T K_{0}^{2} c_{d-1}}{c^{(d-1) / 2}}\right)[\sigma(v)]^{(d-5) / 2}+\sigma(v)\left(\frac{T K_{0}^{2} c_{d}}{c^{d / 2}}\right)[\sigma(v)]^{(d-4) / 2},
\end{aligned}
$$

where the ultraviolet cut-off $\Lambda=2 \pi / a$ has been sent to infinity, and where we defined :

$$
c_{d}=\int \frac{d^{d} \mathbf{k}}{(2 \pi)^{d}} \frac{1}{\left(k^{2}+1\right)^{2}}=\frac{(2-d) \pi^{1-d / 2}}{2^{d+1} \sin (\pi d / 2) \Gamma(d / 2)}
$$

In the limit $L \rightarrow \infty$, the first term on the rhs of equation (89) will be negligibly small compared to the second one, and we can write

$$
1 \simeq \sigma(v)\left(\frac{T K_{0}^{2} c_{d}}{c^{d / 2}}\right)[\sigma(v)]^{(d-4) / 2} .
$$


This approximation will turn out to be valid for a large range of values of the parameter $v$, covering almost the entire interval $[0,1]$ when $L \rightarrow \infty$. Taking the derivative of equation (91) one more time with respect to $v$, and using the fact that $[\sigma]^{\prime}(v)=v \sigma^{\prime}(v)$, we finally obtain ${ }^{13}$

$$
[\sigma](v)=\left(\frac{v}{v_{0}}\right)^{2 / \theta}
$$

where $\theta=d-2$ and $v_{0}=2 T K_{0}^{2} c_{d} /\left((4-d) c^{d / 2}\right)$. Replacing the solution (92) into equation (89), one can verify that the first term on the rhs of that last equation can indeed be neglected if

$$
\left(v_{0} / v\right)^{1 / \theta} \ll L c_{d} / c^{1 / 2} c_{d-1},
$$

which gives, in $d=3$

$$
v \gg \frac{T K_{0}^{2}}{2 \pi L c} .
$$

In the limit $L \rightarrow \infty$, we see that this last condition is satisfied nearly everywhere in the interval $v \in[0,1]$. For films of finite thickness, one can easily show that equations (91)-(92) are still valid provided that the thickness $L$ satisfies

$$
L \gg \frac{T K_{0}^{2}}{2 \pi c} .
$$

a condition which is satisfied by most thin HTSC films even for temperatures close to the superconducting critical temperature $T_{c}$.

The above solution (92) for $[\sigma](v)$ is a priori valid up to a critical value $v_{c}$, above which $[\sigma](v)$ is just a constant, $[\sigma](v)=\left(v_{c} / v_{0}\right)^{2 / \theta}$. With the knowledge of the analytic form of the function $[\sigma](v)$, we now are in a position to find the diagonal part $\tilde{G}\left(\mathbf{q}, q_{n}\right)$ such that

$$
\tilde{C}(\mathbf{x}, z)=\frac{2 T}{L} \sum_{n=-\infty}^{\infty} \int \frac{d^{d-1} \mathbf{q}}{(2 \pi)^{d-1}}\left(1-\cos \left(\mathbf{q} \cdot \mathbf{x}+q_{n} z\right)\right) \tilde{G}\left(\mathbf{q}, q_{n}\right),
$$

and which is given by: ${ }^{14,13}$

$$
\tilde{G}\left(\mathbf{q}, q_{n}\right)=\frac{1}{c\left(q^{2}+q_{n}^{2}\right)}\left(1+\int_{0}^{1} \frac{d v}{v^{2}} \frac{[\sigma](v)}{c\left(q^{2}+q_{n}^{2}\right)+[\sigma](v)}\right) .
$$

Specializing to the three-dimensional case $(d=3)$, if we now use the result (92) with $\theta=d-2=1$ and change the variable of integration to $x=v^{2 / \theta}=v^{2}$, we obtain

$$
\int_{0}^{v_{c}} \frac{d v}{v^{2}} \frac{[\sigma](v)}{c\left(q^{2}+q_{n}^{2}\right)+[\sigma](v)}=\frac{1}{2} \int_{0}^{v_{c}^{2}} \frac{d x}{\sqrt{x}\left(x+c v_{0}^{2}\left(q^{2}+q_{n}^{2}\right)\right)} .
$$

Now, using the result ${ }^{41}$

$$
\int \frac{d x}{\sqrt{x}(x+a)}=\frac{2}{\sqrt{a}} \operatorname{Arctan}(\sqrt{x / a})
$$


we finally obtain

$$
\int_{0}^{v_{c}} \frac{d v}{v^{2}} \frac{[\sigma](v)}{c\left(q^{2}+q_{n}^{2}\right)+[\sigma](v)}=\frac{1}{\sqrt{c v_{0}^{2}}} \frac{1}{\sqrt{q^{2}+q_{n}^{2}}} \operatorname{Arctan}\left(v_{c} / \sqrt{c v_{0}^{2}\left(q^{2}+q_{n}^{2}\right)}\right) .
$$

On the other hand, using the result $[\sigma](v)=\left(v_{c} / v_{0}\right)^{2}$ for $v_{c}<v<1$ it is not difficult to see that

$$
\int_{v_{c}}^{1} \frac{d v}{v^{2}} \frac{[\sigma](v)}{c\left(q^{2}+q_{n}^{2}\right)+[\sigma](v)}=\frac{\left(\frac{1}{v_{c}}-1\right)\left(v_{c} / v_{0}\right)^{2}}{c\left(q^{2}+q_{n}^{2}\right)+\left(v_{c} / v_{0}\right)^{2}} .
$$

Using the fact that $\sqrt{c v_{0}^{2}}=T K_{0}^{2} / 4 \pi c$, and approximating

$$
\operatorname{Arctan}\left(\frac{4 \pi c v_{c}}{T K_{0}^{2} \sqrt{q^{2}+q_{n}^{2}}}\right) \simeq \pi / 2,
$$

(as we are mainly interested in the $\left(\mathbf{q}, q_{n}\right) \rightarrow 0$ behavior of the correlation function), we find, in the long-wavelength limit

$$
\tilde{G}\left(\mathbf{q}, q_{n}\right) \approx\left(\frac{2 \pi^{2}}{T K_{0}^{2}}\right) \frac{1}{\left|q^{2}+q_{n}^{2}\right|^{3 / 2}},
$$

which is nothing but equation (3.18) of reference ${ }^{13}$ in $d=3$. The purpose of the calculation above, equations (84) to (101), was to show that nothing actually changes in Giamarchi and Le Doussal's derivation when we use the decomposition of the $q_{z}$ modes into center of mass and internal modes as we have done throughout this paper, and that the $1 / q^{d}$ behavior of $\tilde{G}\left(\mathbf{q}, q_{n}\right)$ in $d$ dimension previously found by these authors is still valid here. From expression (101), we can easily find the expression of the correlation function $\tilde{C}(\mathbf{x}, z)$. We have:

$$
\tilde{C}(\mathbf{x}, z)=\tilde{C}_{0}(\mathbf{x})+\tilde{C}_{1}(\mathbf{x}, z),
$$

where

$$
\tilde{C}_{0}(\mathbf{x})=\frac{2 T}{L} \int \frac{d^{d-1} \mathbf{q}}{(2 \pi)^{d-1}}\left(1-\cos \left(\mathbf{q} \cdot \mathbf{x}+q_{n} z\right)\right) \tilde{G}(\mathbf{q}, 0),
$$

is restricted to the $\mathrm{CM}$ mode, while

$$
\begin{aligned}
\tilde{C}_{1}(\mathbf{x}, z) & =\frac{2 T}{L} \sum_{n \neq 0} \int \frac{d^{d-1} \mathbf{q}}{(2 \pi)^{d-1}}\left(1-\cos \left(\mathbf{q} \cdot \mathbf{x}+q_{n} z\right)\right) \tilde{G}\left(\mathbf{q}, q_{n}\right), \\
& \simeq \frac{2 T}{L} \int \frac{d^{d} \mathbf{q}}{(2 \pi)^{d}}\left(1-\cos \left(\mathbf{q} \cdot \mathbf{x}+q_{z} z\right)\right) \tilde{G}\left(\mathbf{q}, q_{n}\right)
\end{aligned}
$$

corresponds to the internal modes of the flux lines and is the correlation function calculated by Giamarchi and Le Doussal. ${ }^{13}$ We have, for the CM correlation function $\tilde{C}_{0}(\mathbf{x})$ :

$$
\begin{aligned}
\tilde{C}_{0}(\mathbf{x}) & =\frac{4 \pi^{2} T}{L T K_{0}^{2}} \int \frac{d^{2} \mathbf{q}}{(2 \pi)^{2}} \frac{1-\cos (\mathbf{q} \cdot \mathbf{x})}{q^{3}} \\
& =\frac{2 \pi}{L K_{0}^{2}} \int_{0}^{\Lambda} d q \frac{1-J_{0}(q x)}{q^{2}}
\end{aligned}
$$


Changing variables from $|\mathbf{x}|$ to $u=\Lambda|\mathbf{x}|$, we obtain

$$
\tilde{C}_{0}(\mathbf{x})=\left(\frac{2 \pi}{L K_{0}^{2}}\right)|\mathbf{x}| \times \int_{0}^{\Lambda|\mathbf{x}|} d u \frac{1-J_{0}(u)}{u^{2}}
$$

For large values of $|\mathbf{x}|$, we can to a very good approximation extend the range of integration to infinity. Then, using the fact that ${ }^{26} \int_{0}^{\infty} d u\left(1-J_{0}(u)\right) / u^{2}=1$, we finally obtain

$$
\tilde{C}_{0}(\mathbf{x}) \simeq\left(\frac{2 \pi}{L K_{0}^{2}}\right)|\mathbf{x}|
$$

On the other hand, if we use the result ${ }^{13}$ for $C_{1}(\mathbf{x})$ (in $d=3$ dimensions)

$$
\tilde{C}_{1}(\mathbf{x})=\frac{2}{K_{0}^{2}} \ln |\mathbf{x}|,
$$

we finally obtain

$$
\tilde{C}(\mathbf{x}, z)=\left(\frac{2 \pi}{L K_{0}^{2}}\right)|\mathbf{x}|+\frac{2}{K_{0}^{2}} \ln |\mathbf{x}| .
$$

Now, the translational order correlation function at reciprocal wavevector $\mathbf{K}$

$$
\Psi_{K}(\mathbf{x})=\left\langle\rho_{\mathbf{K}}^{*}(\mathbf{x}) \rho_{\mathbf{K}}(\mathbf{x})\right\rangle
$$

is given by ${ }^{13}$

$$
\Psi_{K}(\mathbf{x})=\mathrm{e}^{-\frac{1}{2} K^{2} \tilde{C}(\mathbf{x})},
$$

which, given the result (108), implies that $C_{K}(\mathbf{x})$ behaves for large $|\mathbf{x}|$ as

$$
\Psi_{K}(\mathbf{x}) \sim \exp \left(-\frac{\pi d_{\perp} K^{2}}{K_{0}^{2}} \frac{|\mathbf{x}|}{L}\right)|\mathbf{x}|^{-\frac{d_{\perp} K^{2}}{2 K_{0}^{2}}}
$$

and hence we see that point disorder does destroy long range order of the FLL, although it does so only on asymptotic length scales $|\mathbf{x}| \sim L$. The same conclusion would follow from more sophisticated functional renormalization-group arguments ${ }^{9,40,15}$.

Equations (106) and (111) are the most important results of this paper. They show clearly that the QLRO of the flux line lattice predicted by considering the full correlation function $C(\mathbf{x}, z)$ and integrating over all internal modes, is actually lost when one carefully separates out the fluctuations of the CM positions of the flux lines. As such, this result confirms our claim that the CM mode plays an important role and is in fact the most relevant one to look at when considering positional order of flux line lattices in samples of highly anisotropic shapes $L \ll L_{\perp}$. For such samples, we expect the destruction of long range range order to be observable in experiments. 


\section{Positional order in the moving flux line lattice in the presence of disorder}

The considerations of the past section can be generalized to the case of a moving FLL in a disordered potential. Here, we shall restrict ourselves to the determination of the dynamic Larkin lengths ${ }^{42}$ for the center of mass positions. Following standard arguments $46,48,42,43,44$, one can show that the usual perturbative expression for the mean-square displacement of a FLL drifting with mean velocity $v$ along the $\mathbf{x}$ direction and subject to a random force $\mathbf{F}(\mathbf{r})$ with the correlations (36);

$$
C(\mathbf{r})=2 W \int \frac{d^{d} \mathbf{q}}{(2 \pi)^{d}} \frac{1-\cos (\mathbf{q} \cdot \mathbf{r})}{\left(\eta v q_{x}\right)^{2}+c^{2} q^{4}},
$$

for a sample of finite thickness $L$ should be replaced by

$$
C(\mathbf{r})=\frac{2 W}{L} \sum_{n} \int \frac{d^{d-1} \mathbf{q}}{(2 \pi)^{d-1}} \frac{1-\cos \left(\mathbf{q} \cdot \mathbf{x}+q_{n} z\right)}{\left(\eta v q_{x}\right)^{2}+c^{2}\left(q^{2}+q_{n}^{2}\right)^{2}} .
$$

In the above expressions, $\eta$ is the microscopic friction coefficient and isotropic elasticity with an elastic constant $c$ is assumed. The mean square displacement of the center of mass mode is given by the $n=0$ term in the above sum, namely

$$
C_{0}(\mathbf{x})=\frac{2 W}{L} \int_{q<\Lambda} \frac{d^{d-1} \mathbf{q}}{(2 \pi)^{d-1}} \frac{1-\cos (\mathbf{q} \cdot \mathbf{x})}{\left(\eta v q_{x}\right)^{2}+c^{2} q^{4}} .
$$

The integral in (114) has been caculated by several authors ${ }^{47,42,43}$. We obtain:

$$
C_{0}(x, \mathbf{y})=\frac{2 W|y|^{4-d}}{\eta L c v} F\left(c x / \eta v y^{2}\right),
$$

where $\mathbf{y}$ represents all the space variables other than $x$ (the direction of the drive) and $z$ (the direction of the magnetic field $\mathbf{B}$ ), and where the scaling function $F$ is such that $F(0)=$ const and $F(x) \sim x^{(3-(d-1)) / 2} \sim x^{(4-d) / 2}$ when $|x| \gg 1$. Defining the dynamical Larkin lengths for the center of mass displacements $R_{0}^{x}$ and $R_{0}^{y}$ by the equation $C\left(R_{0}^{x}, R_{0}^{y}\right) \simeq \xi^{2}$, we obtain:

$$
\begin{aligned}
& R_{0}^{y} \simeq\left(\frac{\eta c v L \xi^{2}}{2 W}\right)^{1 /(4-d)} \\
& R_{0}^{x} \simeq \eta v\left(R_{0}^{y}\right)^{2} / c .
\end{aligned}
$$

Specializing to the case $d=3$, we see that $R_{0}^{y}$ grows only linearly with $v$, in contrast to the transverse Larkin length of the total fluctuations ${ }^{47,42,43,44}$ which exhibits exponential growth as a function of $v, R_{c}^{y} \sim \exp \left(\eta v c \xi^{2}\right) / \Lambda$. The critical lengths $\left(R_{0}^{x}, R_{0}^{y}\right)$ obtained here can however be quite large for macroscopic samples with a large thickness $L$.

\section{Conclusion}

In this article, we have reexamined the problem of the positional order of the Abrikosov flux line lattice in type II superconducting samples of finite thickness 
$L$, carefully separating the center of mass mode of the flux lines from their internal modes. While this separation turns out to be unimportant for clean systems with thermal fluctuations, in the presence of a random pinning potential we find that the Larkin length governing the growth of fluctuations of the center of mass positions is very large for macroscopic samples and grows like $\sqrt{L}$ in $2+1$ dimensions, which suggests that flux line lattices on average retain translational order on length scales much larger than the lengths predicted so far (the latter corresponding to the growth of the internal modes fluctuations of the lines). Going beyond the simple Larkin analysis, within a Gaussian variational approximation with broken replica symmetry we find that translational order of the three dimensional flux line lattice is destroyed, and that the logarithmic roughness predicted in previous work crosses over to power-law growth of the vortex displacements. Although this destruction of positional order only takes place on lateral length scales of order the sample thickness $L$, it can lead to experimentally measurable effects in superconducting thin films.

\section{Appendix A. Eigenvalue of the replicon mode and stability of the replica symmetric solution}

In this appendix we consider the eigenvalue $\lambda$ of the so-called "replicon" mode, which for our problem is given by: ${ }^{14,13}$

$$
\lambda=1-\frac{1}{d_{\perp}} \sum_{\mathbf{K}} K^{4} \Delta_{K} \mathrm{e}^{-\frac{T K^{2}}{L} \sum_{n} \int_{\mathbf{p}} G_{c}\left(\mathbf{p}, p_{n}\right)} \times \frac{1}{L} \sum_{n} \int_{\mathbf{q}} G_{c}^{2}\left(\mathbf{q}, q_{n}\right),
$$

where the connected propagator $G_{c}\left(\mathbf{q}, q_{n}\right)$ is defined by $G_{c}\left(\mathbf{q}, q_{n}\right)=\sum_{b} G_{a b}\left(\mathbf{q}, q_{n}\right)$. Using the saddle point equations (79)-(80), it is not difficult to see that $G_{c}$ is given by $G_{c}\left(\mathbf{q}, q_{n}\right)=1 /\left(c q^{2}+c q_{n}^{2}\right)$. Here, we shall take $G_{c}\left(\mathbf{q}, q_{n}\right)=1 /\left(c q^{2}+c q_{n}^{2}+\mu^{2}\right)$, with a small regularizing mass $\mu^{2}$, the limit $\mu \rightarrow 0$ being taken at the end of the calculation. Transforming the sum over modes $(1 / L) \sum_{n}$ into $q_{z}$ integrals in equation (A.1) right away leads to the conclusion that in $d=2+1$ dimensions the eigenvalue of the replicon mode is always negative, and hence that three dimensional flux line lattices are always unstable to point disorder. Here, we shall instead be careful to separate the $\mathrm{CM}$ from the internal modes, upon which we obtain (here we only consider the case $d=2+1$ dimensions):

$$
\sum_{m} \int_{\mathbf{p}} G_{c}\left(\mathbf{p}, p_{m}\right)=\frac{1}{4 \pi c} \ln \left(1+\frac{\Lambda^{2} c}{\mu^{2}}\right)+\frac{1}{2 \pi c} \sum_{m=1}^{\infty} \ln \left(1+\frac{\Lambda^{2}}{p_{m}^{2}+\mu^{2} / c}\right),
$$

and so, in the limit $\mu \rightarrow 0$ we can write:

$$
\left.\frac{1}{L} \sum_{m} \int_{\mathbf{p}} G_{c}\left(\mathbf{p}, p_{m}\right)\right|_{\mu \rightarrow 0}=\frac{1}{4 \pi L c} \ln \left(\frac{\Lambda^{2} c}{\mu^{2}}\right)+C_{1},
$$

where $C_{1}$ is the constant given by

$$
C_{1} \simeq \frac{1}{2 \pi c} \int_{0}^{\infty} \frac{d q_{z}}{2 \pi} \ln \left(1+\Lambda^{2} / q_{z}^{2}\right)
$$


On the other hand, one can also easily show that

$$
\left.\frac{1}{L} \sum_{m} \int_{\mathbf{p}} G_{c}^{2}\left(\mathbf{p}, p_{m}\right)\right|_{\mu \rightarrow 0}=\frac{1}{4 \pi L c^{2} \mu^{2}}+C_{2},
$$

where

$$
C_{2}=-\frac{1}{4 \pi L c^{2} \Lambda^{2}}+\frac{1}{2 \pi L c^{2}} \sum_{n \geq 1}\left(\frac{1}{q_{n}^{2}}-\frac{1}{q_{n}^{2}+\Lambda^{2}}\right) .
$$

Collecting all terms, we see that $\lambda$ can be written as

$$
\lambda=1-\frac{1}{d_{\perp}} \sum_{\mathbf{K}} K^{4} \Delta_{K} \mathrm{e}^{-T K^{2} C_{1}}\left(\frac{\Lambda^{2} c}{\mu^{2}}\right)^{-T K^{2} / 4 \pi L c} \times\left[\frac{1}{4 \pi L c^{2} \mu^{2}}+C_{2}\right] .
$$

In the limit $\mu \rightarrow 0$, the dominant term in the above sum is the term with $K=K_{0}$, which gives:

$$
\lambda \approx 1-\frac{K_{0}^{4} \Delta_{K_{0}}}{4 \pi L d_{\perp} c^{2}} \mathrm{e}^{-T K_{0}^{2} C_{1}}\left(\Lambda^{2} c\right)^{-\frac{T K_{0}^{2}}{4 \pi L c}} \mu^{-2\left(1-\frac{T K_{0}^{2}}{4 \pi c}\right)} .
$$

From this last expression, we see that the sign of $\lambda$ when $\mu \rightarrow 0$ depends on the value of $T K_{0}^{2} / 4 \pi L c$ :

- For $T K_{0}^{2} / 4 \pi L c>1$, i.e. $T>T_{c}=4 \pi L c / K_{0}^{2}, \lambda(\mu \rightarrow 0)=1>0$, the replicasymmetric solution is stable;

- For $T K_{0}^{2} / 4 \pi L c<1$, or $T<T_{c}=4 \pi L c / K_{0}^{2}, \lambda(\mu \rightarrow 0) \rightarrow-\infty$ and the replica symmetric solution is unstable.

An identical result has been obtained by Giamarchi and Le Doussal in ref. ${ }^{20}$ (where the authors adopted a separation of modes similar to ours) in the context of correlated disorder. We thus see that the conclusions of this last reference regarding the stability of the replica symmetric solution result solely from the separation of modes into CM and internal modes, and has nothing to do with the nature of the (point-like or correlated) disorder considered.

\section{References}

1. A.A. Abrikosov, Sov. Phys. JETP 5, 1174 (1957).

2. J.G. Bednorz and K.A. Muller, Z. Phys. 64, 189 (1986).

3. G. Blatter, M.V. Feigel'man, V.B. Geshkenbein, A.I. Larkin and V.M. Vinokur, Rev. Mod. Phys.66, 1125 (1994).

4. T. Nattermann and S. Scheidl, Advances in Physics 49, 607 (2000).

5. A.I. Larkin, Sov. Phys. JETP 31, 784 (1970).

6. Y. Imry and S.K. Ma, Phys. Rev. Lett. 35, 1399 (1975).

7. P.A. Lee and T.M. Rice, Phys. Rev. B 19, 3970 (1979).

8. J. Villain and Fernandez, Z. Phys. B54, 139 (1984); and references therein.

9. D.S. Fisher, Phys. Rev. Lett. 56, 1964 (1986).

10. T. Nattermann, Phys. Rev. Lett. 64, 2454 (1990).

11. J.-P. Bouchaud, M. Mézard and J.S. Yedidia, Phys. Rev. Lett. 67, 3840 (1991); Phys. Rev. B 4614686 (1992).

12. S.E. Korshunov, Phys. Rev. B 48, 3969 (1993). 
13. T. Giamarchi and P. Le Doussal, Phys. Rev. Lett. 72, 1530 (1994); Phys. Rev. B 52, $1242(1995)$.

14. M. Mézard and G. Parisi, J. Phys I (France) 1, 809 (1991).

15. T. Emig, S. Bogner and T. Nattermann, Phys. Rev. Lett. 83, 400 (1999).

16. D.S. Fisher, Phys. Rev. B 22, 1190 (1980).

17. A.I. Larkin and Yu.N. Ovchinnikov, Sov. Phys. JETP 38, 854 (1974).

18. See for example M. Doi and S.F. Edwards, The Theory of Polymer Dynamics, Oxford, (1986).

19. L. Radzihovsky and E. Frey, Phys. Rev. B 48, 10357 (1993).

20. T. Giamarchi and P. Le Doussal, Phys. Rev. B 53, 15206 (1996).

21. E.H. Brandt, J. Low Temp. Phys. 26, 709 (1977); 26, 735 (1977); 28, 263 (1977); 28, 291 (1977).

22. P.M. Chaikin and T.C. Lubensky, Principles of Condensed Matter Physics, Cambridge University Press, (1995).

23. This constant actually depends on the cut-off procedure. For a square lattice, it is given by $\gamma+\frac{1}{2} \ln 8$, where $\gamma \simeq 0.5772$ the Euler-Mascheroni constant (see ref. $^{22}$ ).

24. This logarithmic behaviour of the CM relative displacement in pure samples has already been noticed by some authors; see for example H. Nordborg and G. Blatter, Phys. Rev. B58, 14556 (1998).

25. P.G. de Gennes, Superconductivity of Metals and Alloys, Perseus Books, (1966).

26. M. Abramowitz and I.A. Stegun, Handbook of Mathematical Functions, Dover (1972).

27. V.L. Pokrovsky and A.L. Talapov, Phys. Rev. Lett. 42, 65 (1979).

28. A.A. Abrikosov, Sov. Phys. JETP 19, 988 (1964).

29. E.H. Brandt, J. Low Temp. Phys. 64, 375 (1986).

30. E. Chudnovsky, Phys. Rev. B 43, 7831 (1991).

31. Note that the Hamiltonian (38) can be diagonalized exactly without minimization (using replicas to average over the random field), in which case one obtains an additional, subdominant contribution to the correlation functions in equations (41) and (44). Here we neglect this thermal part and focus on the dominant random force contributions.

32. P.H. Kes and R. Wördenweber, J. Low Temp. Phys. 67, 1 (1987).

33. D.R. Nelson and V.M. Vinokur, Phys. Rev B 48, 13060 (1993).

34. F.D.M. Haldane, Phys. Rev. Lett. 47, 1840 (1981).

35. M.P.A. Fisher, Phys. Rev. Lett. 62, 1415 (1989).

36. R.P. Feynman, Statistical Mechanics: A Set of Lectures, Perseus Books, 1998.

37. J.R.L. de Almeida and D.J. Thouless, J. Phys. A11, 983 (1978).

38. G. Parisi, J. Phys. A13, 1101 (1980).

39. For further details on how this parametrization emerges in the replica-broken phase in the limit $n \rightarrow 0$, we refer the reader to ${ }^{45}$ and references therein.

40. L. Balents and D.S. Fisher, Phys. Rev. B 48, 5949 (1993).

41. A.P. Prudnikov, Yu. A. Brychkov and O.I. Marichev, Integrals and Series, New York, Gordon and Breach, 1986.

42. T. Giamarchi and P. Le Doussal, Phys. Rev. Lett. 76, 3408 (1996); Phys. Rev. B 57, 11356 (1998).

43. L. Balents, M.C. Marchetti and L. Radzihovsky, Phys. Rev. Lett. 78, 751 (1997); Phys. Rev. B 57, 7705 (1998).

44. S. Scheidl and V.M. Vinokur, Phys. Rev. B 57, 13800 (1998).

45. V. Dotsenko, An introduction to the theory of spin glasses and neural networks, World Scientific, 1994.

46. L. Sneddon, M.C. Cross and D.S. Fisher, Phys. Rev. Lett. 49, 292 (1982).

47. L. Radzihovsky and J. Toner, Phys. Rev. Lett. 81, 3711 (1998). 
November 20, 2018 1:5 WSPC/INSTRUCTION FILE paper

Positional order of the pinned Abrikosov vortex lattice in samples of finite thickness

48. L. Balents and M.P.A. Fisher, Phys. Rev. Lett. 75, 4270 (1995). 